\title{
An EAS-like event registered with the TUS orbital detector
}

\section{G.K. Garipov, M.A. Kaznacheeva, B.A. Khrenov, P.A. Klimov, M.I. Panasyuk,} V.L. Petrov, S.A. Sharakin, A.V. Shirokov, I.V. Yashin, M.Yu. Zotov

Lomonosov Moscow State University, Skobeltsyn Institute of Nuclear Physics, Moscow, 119991, Russia

\section{V.M. Grebenyuk, A.A. Grinyuk, M.V. Lavrova, A.V. Tkachenko, L.G. Tkachev}

Joint Institute for Nuclear Research, Joliot-Curie, 6, Dubna, Moscow region, Russia, 141980

\section{A.A. Botvinko, O.A. Saprykin, A.E. Puchkov, A.N. Senkovsky}

Space Regatta Consortium, ul. Lenina, 4a, 141070 Korolev, Moscow region, Russia

\section{and M. Bertaina*}

Università degli studi di Torino, Via Pietro Giuria 1, 10125 Turin, Italy

E-mail: bertaina@to.infn.it

\begin{abstract}
TUS (Tracking Ultraviolet Set-up) is the world's first orbital detector of ultra-high-energy cosmic rays (UHECRs). It was launched into orbit on April 28, 2016, as a part of the scientific payload of the Lomonosov satellite. The main aim of the mission was to test the technique of measuring the ultraviolet fluorescence and Cherenkov radiation of extensive air showers generated by primary cosmic rays with energies above $\sim 100 \mathrm{EeV}$ in the nocturnal atmosphere of the Earth from space. During its operation period, TUS registered almost 80,000 events, with a few of them satisfying the criteria expected for UHECR candidate events. Here we discuss the phenomenology and possible interpretations of an outstanding event registered in perfect observational conditions on October 3, 2016.
\end{abstract}

36th International Cosmic Ray Conference -ICRC2019-

July 24th - August 1st, 2019

Madison, WI, U.S.A.

${ }^{*}$ Speaker. 


\section{Introduction}

Measurements of the energy spectrum, nuclear composition and arrival directions of ultrahigh-energy cosmic rays (UHECRs, $E \gtrsim 50 \mathrm{EeV}^{1}$ ) are an important part of modern astrophysics and particle physics [1] but the ongoing studies are limited by the very low flux of the particles. The primary goal of TUS, the world's first orbital detector of UHECRs [2, 3], was to expand the experimental studies of UHECRs to space following an idea by Benson and Linsley [4]. The main point is that fluorescence and Cherenkov ultraviolet (UV) radiation of an extensive air shower (EAS) generated by an UHECR in the nocturnal atmosphere of the Earth can be detected from a satellite similar to the way it is observed from the ground with fluorescence telescopes but with a much larger and uniform exposure of the whole celestial sphere.

The TUS instrument on board the Lomonosov satellite was launched into orbit from the newly built Vostochny Cosmodrome (Russia) on April 28, 2016, and successfully operated till late 2017. Several preliminary UHECR candidates have been selected in the mission data [5] and are being analyzed. In the present work, we focus on an event registered on October 3, 2016 (TUS161003 for short), which is remarkable due to the shape and kinematics of the signal closely resembling what is expected from an EAS. We discuss the event and related puzzles and comment on its astrophysical and anthropogenic aspects.

\section{The TUS detector}

TUS was a UV telescope looking downward into the atmosphere in the nadir direction. Its design was determined by the task of registering fluorescence and backscattered (reflected) Cherenkov radiation of EASs in the near-UV band. The two main parts of the instrument were a modular Fresnel mirror-concentrator and 256 photomultiplier tubes (PMTs) arranged in a $16 \times 16$-channels photodetector located in the focal plane of the mirror.

The TUS mirror was composed of 7 hexagonal segments made of a carbon plastic with the total area of $S_{\text {mirr }}=1.93 \mathrm{~m}^{2}$ and the focal distance of $1500 \mathrm{~mm}$. The overall field of view (FOV) of the detector was $9^{\circ} \times 9^{\circ}$.

The photodetector was built of 16 modules, each consisting of 16 channels. Each channel (pixel) was a Hamamatsu R1463 PMT with the quantum efficiency of approximately $20 \%$ in the wavelength band $300-400 \mathrm{~nm}$. A multi-alcali cathode was covered by a UV glass filter of the UFS 1 type and a reflective light guide with a square entrance of $15 \mathrm{~mm}$ size located in the focal plane of the mirror. The angular resolution (the FOV of one channel) was $10 \mathrm{mrad}$, which corresponds to a spatial domain of about $5 \mathrm{~km} \times 5 \mathrm{~km}$ at the sea level from a $500 \mathrm{~km}$ orbit height. Thus, the full instantaneous area observed by TUS was approximately $80 \mathrm{~km} \times 80 \mathrm{~km}=6400 \mathrm{~km}^{2}$.

TUS employed a two-level trigger [6], which was able to select events in terms of both the intensity of the signal (a threshold trigger) and the specific space-time pattern (an adjacency trigger). Data of all 256 channels for 256 consecutive time steps with a time resolution of $0.8 \mu$ s were recorded in case conditions of both triggers were satisfied, thus forming a TUS event. ${ }^{2}$

\footnotetext{
${ }^{1} 1 \mathrm{EeV}=10^{18} \mathrm{eV}$

${ }^{2}$ TUS also performed observations with sampling times of $25.6 \mu \mathrm{s}, 0.4 \mathrm{~ms}$ and $6.6 \mathrm{~ms}$ for registering slower UV phenomena.
} 
The Lomonosov satellite had a sun-synchronous orbit with an inclination of $97.3^{\circ}$, a period of $\approx 94 \mathrm{~min}$, and a height of about $470-500 \mathrm{~km}$ above the sea level. A more detailed description of the TUS detector can be found in [2]. Preliminary results of the TUS mission were presented in $[7,3,8]$.

\section{The TUS161003 event}

The TUS161003 event was registered on October 3, 2016, at 05:48:59 UTC, 00:48:59 local time. The center of the FOV of TUS at the moment of registration was located at $44.08^{\circ} \mathrm{N}, 92.71^{\circ} \mathrm{W}$ above Minnesota, USA. The orbit height was about $481 \mathrm{~km}$ above the sea level.

Position of the FOV of TUS on the ground at the moment of registration of the event is shown in the left panel of Fig. 1. The cyan arrow shows the approximate direction of movement of the signal. There are no big cities, airports or other obvious potential sources of the signal below the hit channels.
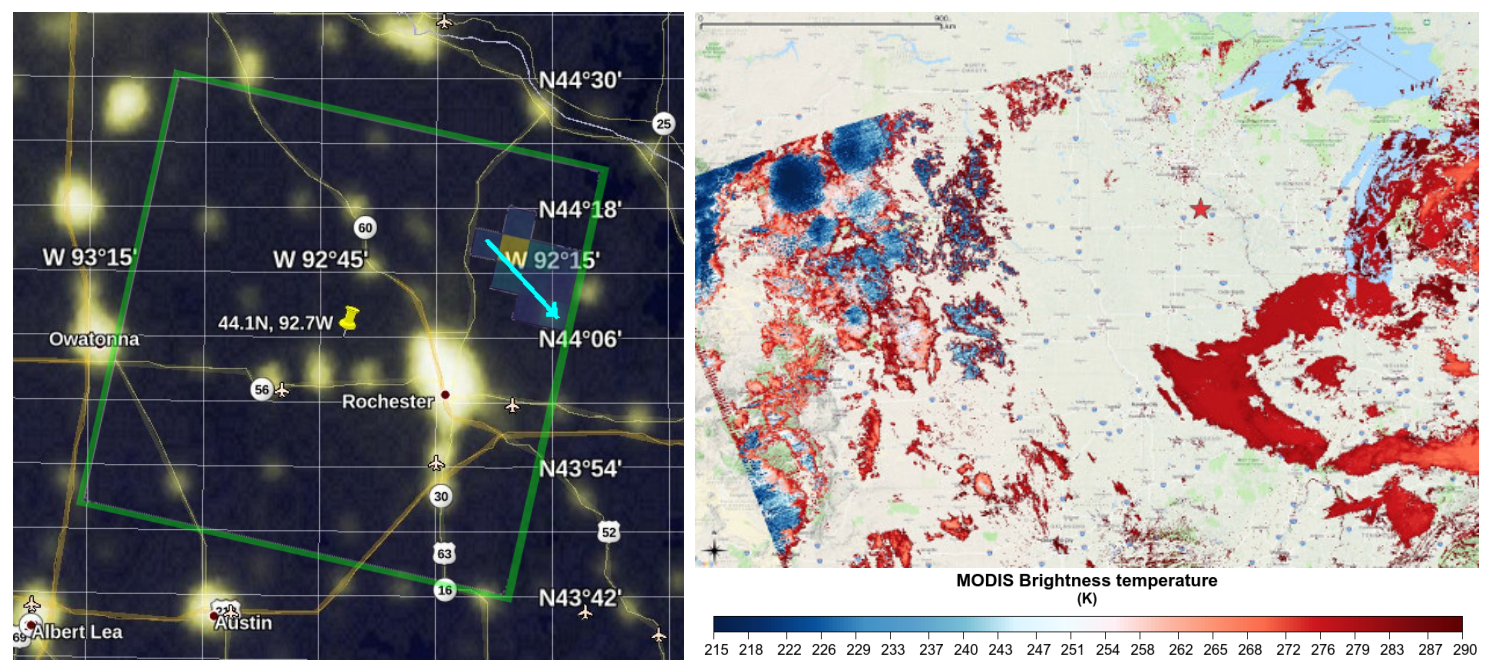

Figure 1: Left panel: the FOV of TUS indicated by green lines for the TUS161003 event on a Google Earth ${ }^{4}$ map with the NASA data on night Earth. Right panel: MODIS data on the cloud coverage near the time and region of registration of the event. The star shows the location of the event.

A detailed analysis of conditions of observation was performed to exclude possible atmospheric sources that could imitate an EAS signal. Thunderstorm activity was studied in the region using the Vaisala Global Lightning Dataset GLD360, a ground-based lightning location network with a relatively high detection efficiency $[9,10]$. No lightning strikes were registered within $930 \mathrm{~km}$ and during $\pm 10 \mathrm{~s}$ from the event. This witnesses in favour of a non-thunderstorm origin of the signal.

A study of the cloud coverage was performed using the MODIS ${ }^{5}$ satellite images and information from the Modern-Era Retrospective analysis for Research and Applications-2 (MERRA2) project. ${ }^{6}$ The cloud coverage in the region of the event provided by MODIS is shown in the right

\footnotetext{
${ }^{4}$ https://www.google.com/earth/

${ }^{5}$ https://modis.gsfc.nasa.gov/

${ }^{6}$ https://gmao.gsfc.nasa.gov/reanalysis/MERRA-2/
} 
panel of Fig. 1. The colours correspond to the brightness temperatures of clouds. The position of the event is marked by a star. The transparent area indicates the absence of clouds. It can be seen that the atmosphere was clear at the time of observation of the event without any noticeable clouds except some small low-altitude ones.

Waveforms for ten hit channels found in the TUS161003 event are shown in Fig. 2. The average level of the background illumination is extracted from the ADC codes for clarity. The waveforms are approximated with a so-called bi-Gaussian function, i.e., two Gaussian functions smoothly sewed at the point of the maximum. The waveforms are ordered according to the position of the maximum of the fits, from top to bottom beginning from the left column. The hit channels are grouped in an oblong spot, the shape of which might be a "convolution" of two factors, namely, the asymmetric PSF of the mirror and a linear track. Moments of the maximum of the signal approximation in each channel have some shift from one channel to another, which can be interpreted as a movement of the signal in the FOV of the detector.
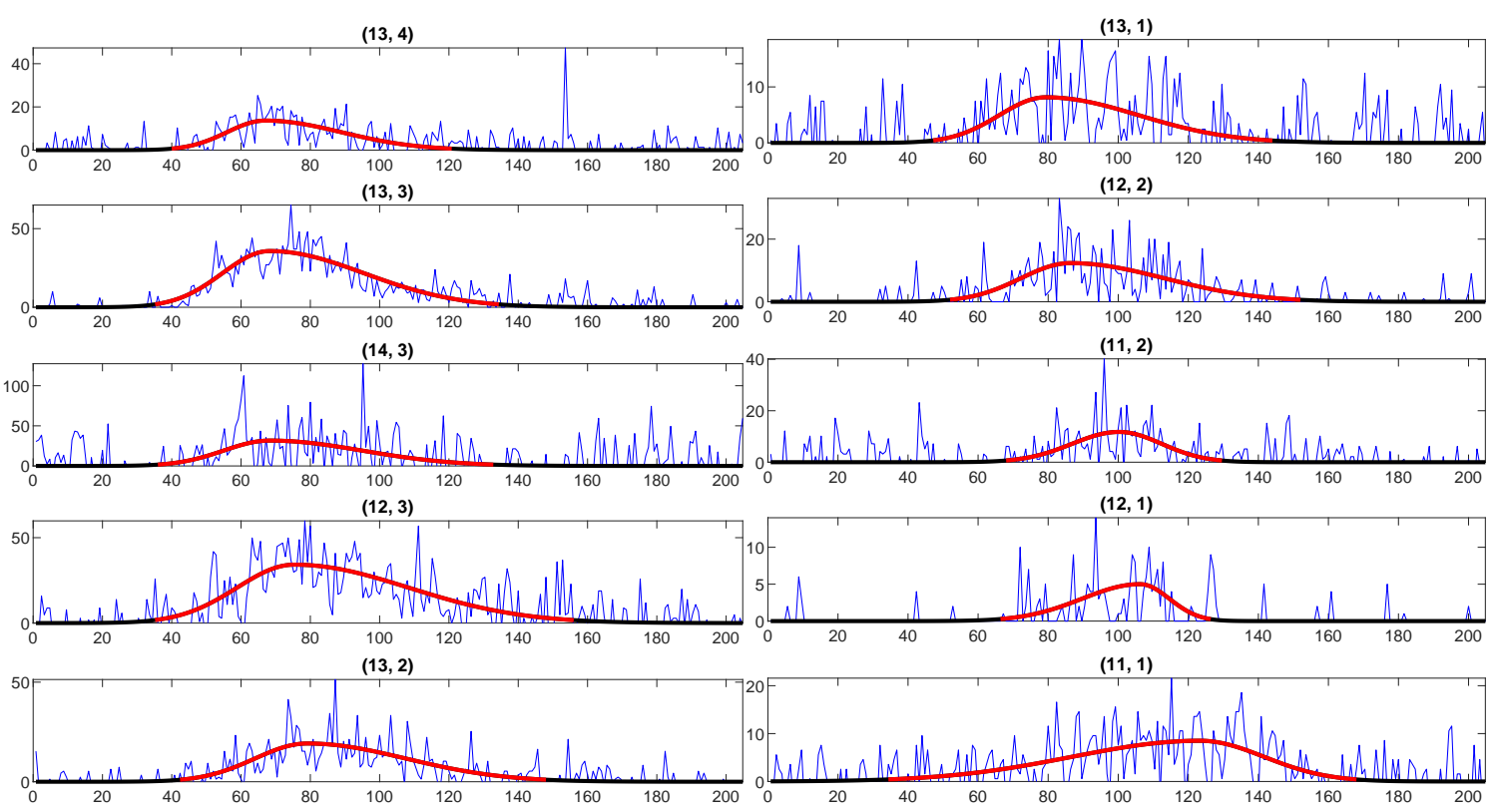

Figure 2: Signals in ten hit channels of the TUS161003 event with bi-Gaussian approximations. The average level of background illumination is extracted from the signals for clarity. Numbers in parenthesis above the panels indicate the module and channel numbers respectively. The $x$-axes represent time in $\mu \mathrm{s}$.

The light curve of the event is shown in Fig. 3. The curve is clearly asymmetric, which is most likely due to the delayed signal as a result of multiple scattering of photons in the atmosphere. The following set of parameters was obtained by fitting the light curve with a bi-Gaussian function with a coefficient of asymmetry defined as a ratio of the attenuation time to the rise time:

- the amplitude of the signal $I_{\max } \approx 214$ ph. $\mu \mathrm{s}^{-1} \mathrm{~m}^{-2}$;

- full duration at half maximum FDHM $\approx 58 \mu \mathrm{s}$;

- the coefficient of asymmetry $\alpha \approx 2.4$. 


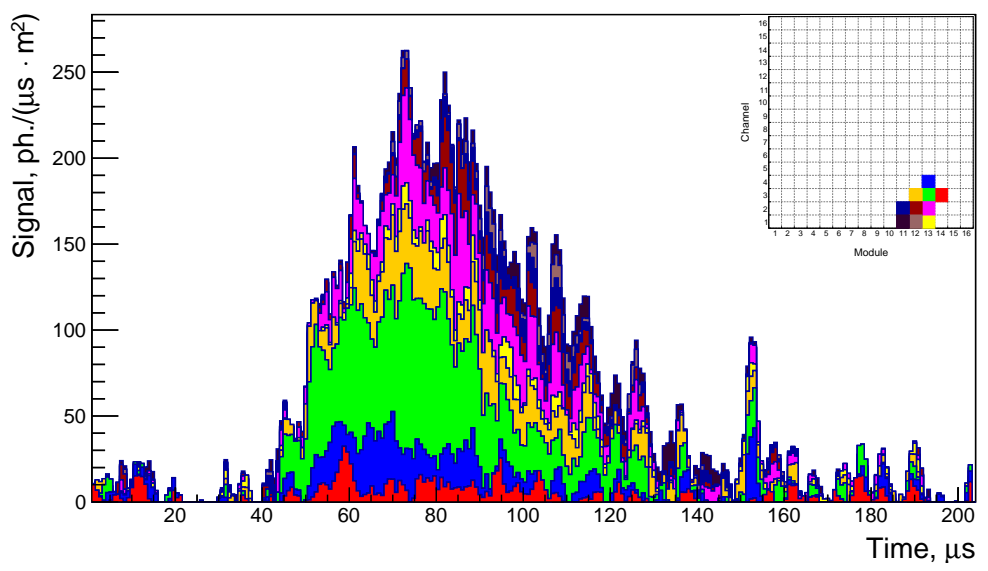

Figure 3: Light curve of the TUS161003 event as the sum of signals in the ten hit channels. The signal is smoothed with a moving average calculated for three time steps to minimize statistical fluctuations. The average background illumination was extracted. The inset shows positions of the hit pixels in the focal surface.

\section{Reconstruction of the signal parameters}

A reconstruction of the arrival direction of a relativistic particle can be seen as a search for parameters of its track, i.e., a line segment on the focal plane of the detector, along which the signal moves at a constant velocity:

$$
x(t)=x_{0}+u_{x}\left(t-t_{0}\right), \quad y(t)=y_{0}+u_{y}\left(t-t_{0}\right),
$$

where $x(t)$ and $y(t)$ are Cartesian coordinates of the image center in the focal plane at time $t,\left(x_{0}, y_{0}\right)$ is a point on the track corresponding to some $t=t_{0}$, and $u_{x}, u_{y}$ are projections of the point velocity on the axes of the local Cartesian coordinate system. However, one should keep in mind that an image of a point source hardly ever occupies a single channel because of the PSF, and the track is not exactly linear because of the discrete structure of the focal plane.

An heuristic method of estimating parameters of a track was suggested in [11]. The method is based on minimizing weighted least squares for Eqs. (4.1), independently for $x$ and $y$. Weights are chosen depending on signal values in the hit channels. An analysis that followed revealed the method was sensitive to the choice of hit channels in case some of them have a weak signal. The method was modified to diminish the effect. In the new algorithm, one minimizes the sum

$$
\sum_{i} \sum_{k=k_{1}(i)}^{k_{2}(i)} W_{i}\left(t_{k}\right) \cdot\left(x_{0}+t_{k} u_{x}-X_{i}\right)^{2}
$$

over parameters $x_{0}$ and $u_{x}$, and a similar sum for $y_{0}, u_{y}$ and $Y_{i}$. Here, an index $i$ runs over all hit channels in the event, $W_{i}\left(t_{k}\right)$ is a weight proportional to the value of the signal at time $t_{k}$, and $X_{i}$ and $Y_{i}$ are coordinates of the center of the $i$ th pixel.

One of the key modifications aimed at reducing the influence of statistical fluctuations of the ADC code on the results of the reconstruction is a search for the moments $t_{k}$ when the $i$ th signal is "active." It was shown in a dedicated study of simulated events that the choice of the threshold for 
selecting an activity window equal to $30 \%$ relative to the amplitude value makes the reconstruction procedure more robust. $^{7}$

The accuracy of the method in application to the TUS161003 event was studied by simulating 1000 events with azimuth angles $\phi=40^{\circ} \ldots 60^{\circ}$, zenith angles $\theta=35^{\circ} \ldots 55^{\circ}$ and the subsequent reconstruction of the initial parameters. As a result, the following estimates of the arrival direction of the TUS event were obtained assuming it was caused by a relativistic particle:

$$
\phi=\left(50_{-10}^{+3}\right)^{\circ}, \quad \theta_{\mathrm{rec}}=(44 \pm 4)^{\circ} .
$$

An energy of a primary particle that presumably initiated the event is another parameter to be found. One needs to know the sensitivity of PMTs forming the focal surface to accomplish the task. Unfortunately, the system of automatic adjustment of high voltage did not work properly during the first day of TUS operating in orbit. This lead to the loss of nearly $20 \%$ of all PMTs and a change of sensitivities of the rest. A special procedure for an in-flight calibration of the working channels was developed to overcome the problem. The method relies on an analysis of stationary waveforms in the main mode of operation and in the one with the longest time steps. A thorough application of the procedure to the ten hit channels, which will be explained in details elsewhere, allowed us to estimate their new sensitivities. Together with extensive simulations of the detector response to EASs generated by primary particles in the energy range $100-1100 \mathrm{EeV}$ performed with ESAF [12, 13], this took us to the lower limit of the energy of an UHECR $\approx 1000 \pm 200 \mathrm{EeV}$.

Figure 4 illustrates an expected response of TUS to an EAS generated by a $1 \mathrm{ZeV}$ proton arriving at the zenith angle $\theta \approx 50^{\circ}$. Five orders of scattering of fluorescence and Cherenkov photons in the atmosphere were taken into account. An albedo of the surface was set equal to $2 \%$ since this is the value of albedo of grass in the UV range of 320-390 nm [14], and the TUS161003 event was registered above a similar surface. One can see the dynamics of the signal similar to the one shown in Fig. 2 for the TUS161003 event. The light curves demonstrate similar amplitudes but the attenuation time in the simulated event is considerably shorter than in the real one due to the shower front hitting the ground.

\section{Discussion}

It is straightforward to estimate the angular velocity of the image of the TUS161003 event in the focal surface as $\omega \approx 262 \mathrm{rad} / \mathrm{s}$. This corresponds to the apparent velocity $\approx 0.43 \mathrm{c}$ of an object moving at the distance of $500 \mathrm{~km}$ from the detector (perpendicular to the line of site). This allows us to conclude that TUS registered a signal moving at a relativistic speed. This also justifies the application of the method for reconstructing the arrival direction described above. A separate analysis revealed that the signal was moving downward to the ground.

The most natural candidate to explain such a signal is an EAS generated by an UHECR. However, the estimates presented above demonstrate a primary particle should have an energy of the order of $1 \mathrm{ZeV}$ to provide a signal with an amplitude similar to that in the TUS161003 event. No CRs with so extreme energies have ever been registered with ground-based experiments. Taking

\footnotetext{
${ }^{7}$ Another parameter controlling the work of the method is the exponent $n$ of weights $W_{i}$. It turned out that the smallest bias in the reconstruction takes place for $n \approx 1$, thus the exponent is omitted in 4.2.
} 

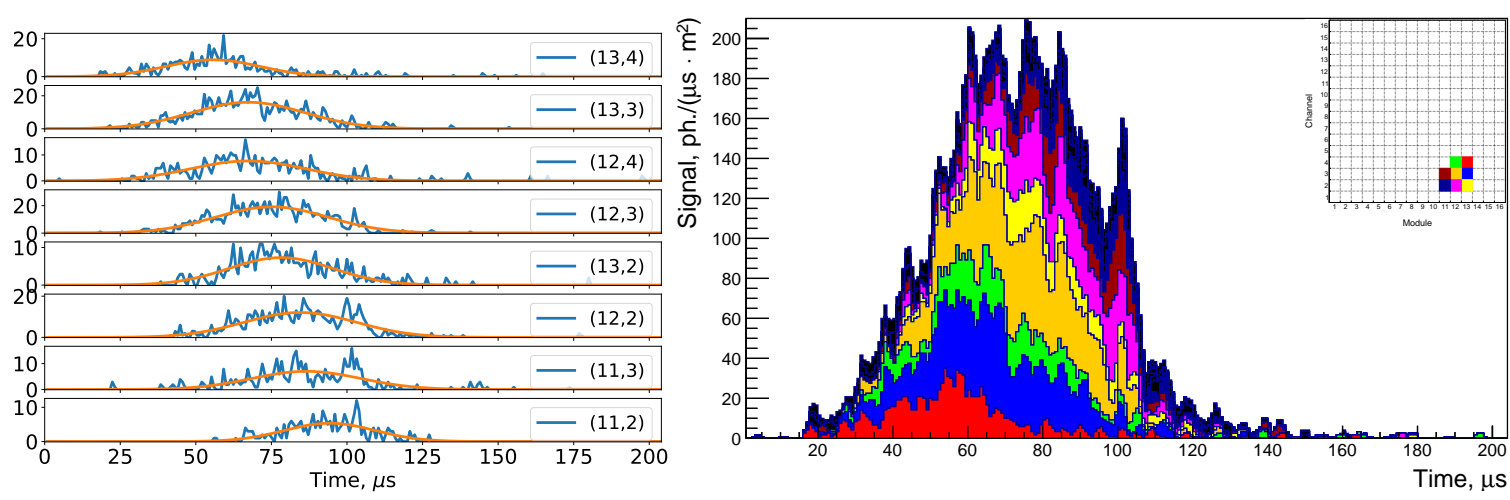

Figure 4: The TUS detector response to an EAS generated by a $1 \mathrm{ZeV}$ proton arriving at $\theta \approx 50^{\circ}$. Left: ADC codes of eight hit channels. Right: the light curve. The inset shows positions of the hit pixels in the focal surface.

into account a limited exposure of the TUS mission ( $\sim 1200-1400 \mathrm{~km}^{2} \mathrm{sr}$ yr as the first extimation) and the fact that the flux of $\mathrm{ZeV} \mathrm{CRs}$ is expected to be 2-4 orders of magnitude lower than that around $100 \mathrm{EeV}$, chances of TUS registering such an energetic cosmic particle are very low.

Another important phenomenological feature of the event is that it develops very high in the atmosphere. The duration of the signal and its slow attenuation $\sim 60 \mu$ s clearly seen in Fig. 3 allow one to estimate $X_{\max } \approx 550 \mathrm{~g} / \mathrm{cm}^{2}$, which corresponds to the altitude of $\sim 7.5 \mathrm{~km}$. An even higher altitude of the signal maximum is obtained if one assumes the narrow peak at around $150 \mu$ s to be the Cherenkov one. These values are in a contradiction with what one expects from a $\mathrm{ZeV}$ event.

Interestingly, relativistic dust grains $[15,16]$ suggest a possible explanation of an extremeenergy event developing high in the atmosphere. According to simulations [17, 18], an EAS initiated by a massive relativistic dust grain develops at $X_{\max } \sim 200-400 \mathrm{~g} / \mathrm{cm}^{2}$, which seemingly agrees with the TUS161003 event. Remarkably, a cascade reaching its maximum high in the atmosphere can explain why ground-based fluorescent detectors do not observe such events.

Finally, one should consider an anthropogenic origin of the TUS161003 event since its thunderstorm origin is unlikely. One of the possibilities consistent with the signal moving downward is a laser shooting from the height of a few kilometers, for example, from an airplane. However, simple simulations show that the observed light curve cannot be explained by a laser lidar operation in a pulse mode since the light curve should demonstrate either an exponential decay or a sharp cut-off as soon as the beam hits the ground.

\section{Acknowledgments}

We thank Francesco Fenu and Kenji Shinozaki for their invaluable help with ESAF and many other related questions, and Roberto Cremonini for the cloud coverage analysis. We also thank Vaisala Inc. company for providing the relevant data on lightning strikes. The work was done with partial financial support from the State Space Corporation ROSCOSMOS, M.V. Lomonosov Moscow State University through its "Prospects for Development" program and the Russian Foundation for Basic Research grant No. 16-29-13065 and 15-02-05498/17-a. The Italian group acknowledges financial contribution from the agreement ASI-INAF n.2017-14-H.O. 


\section{References}

[1] B. R. Dawson, M. Fukushima, and P. Sokolsky, Past, present and future of UHECR observations, Prog. Theor. Exp. Phys., 12 (2017) 12A101 [1703.07897].

[2] P. A. Klimov, M. I. Panasyuk, B. A. Khrenov, et al., The TUS detector of extreme energy cosmic rays on board the Lomonosov satellite, Space Science Reviews, 8 (2017) 1 [1706 . 04976 ].

[3] B. A. Khrenov, P. A. Klimov, M. I. Panasyuk, et al., First results from the TUS orbital detector in the extensive air shower mode, J. of Cosmology and Astroparticle Physics, 09 (2017) 006 [1704.07704].

[4] R. Benson and J. Linsley, Satellite observation of cosmic-ray air showers, In Bulletin of the American Astronomical Society, 12 (1980) 818.

[5] S. V. Biktemerova, A. A. Botvinko, N. P. Chirskaya, et al., Search for extreme energy cosmic ray candidates in the TUS orbital experiment data, [arXiv:1706.05369].

[6] A. Grinyuk, V. Grebenyuk, B. Khrenov, et al., The orbital TUS detector simulation, Astroparticle Physics, 90 (2017) 93.

[7] M. Zotov for the Lomonosov UHECR/TLE collaboration, Early results from TUS, the first orbital detector of extreme energy cosmic rays, in 2016 Conference on Ultrahigh Energy Cosmic Rays (UHECR2016) Kyoto, Japan, October 11-14, 2016, (2018) 011029 [1703.09484].

[8] M. Zotov for the Lomonosov UHECR/TLE collaboration, First results of the TUS orbital detector of extreme-energy cosmic rays, Physics of Particles and Nuclei, 49 (2018) 612.

[9] R. K. Said, U. S. Inan, and K. L. Cummins, Long-range lightning geolocation using a VLF radio atmospheric waveform bank, Journal of Geophysical Research (Atmospheres), 115 (2010) D23108.

[10] R. Said and M.J. Murphy, GLD360 upgrade: Performance analysis and applications, in 24th Int. Lightning Detection Conf. and Sixth Int. Lightning Meteorology Conf., American Institute of Physics Conference Series, (2016) 8.

[11] L. Tkachev and Lomonosov-UHECR/TLE Collaboration, Search for EAS candidates with the TUS/Lomonosov orbital experiment: results of preliminary data reconstruction and analysis, in Proceedings of Science (ICRC2017), (2017) 527.

[12] C. Berat, S. Bottai, D. De Marco et al., Full simulation of space-based extensive air showers detectors with ESAF, Astroparticle Physics, 33 (2010) 221.

[13] M. Bertaina, A. Castellina, R. Cremonini et el., Search for extreme energy cosmic rays with the TUS orbital telescope and comparison with ESAF, in EPJ Web of Conferences, 210 (2019) 06006.

[14] R. Chadyšienė and A. Girgždys, Ultraviolet radiation albedo of natural surfaces, J. of Environmental Engineering and Landscape Management, 16 (2008) 83.

[15] L. Spitzer, On the origin of heavy cosmic-ray particles, Phys. Rev., 76 (1949) 583.

[16] S. Hayakawa, Dust grain origin of cosmic ray air showers, Astrophysics and Space Science, 16 (1972) 238.

[17] L. Anchordoqui, Cosmic dust grains strike again, Phys. Rev. D, 61 (2000) 087302.

[18] B. A. Khrenov, M. I. Panasyuk, V. V. Alexandrov, et al., Space Program KOSMOTEPETL (project KLYPVE and TUS) for the study of extremely high energy cosmic rays, in Observing Ultrahigh Energy Cosmic Rays from Space and Earth, American Institute of Physics Conference Series, 566 (2001) 57. 